\title{
Conductance modulation by spin precession in noncollinear ferromagnet normal-metal ferromagnet systems
}

\author{
Daniel Huertas Hernando, ${ }^{1}$ Yu. V. Nazarov, ${ }^{1}$ Arne Brataas, ${ }^{2}$ and Gerrit E. W. Bauer ${ }^{1}$ \\ ${ }^{1}$ Department of Applied Physics and Delft Institute of Microelectronics and Submicrontechnology, Delft University of Technology, \\ Lorentzweg 1, 2628 CJ Delft, The Netherlands \\ ${ }^{2}$ Harvard University, Lyman Laboratory of Physics, Cambridge, Massachusetts 02138
}

(Received 23 March 2000)

\begin{abstract}
We study diffusive transport through ferromagnet normal-metal ferromagnet systems, with arbitrary but fixed magnetization directions of the ferromagnetic reservoirs and orientations of a magnetic field applied to the normal metal. For noncollinear configurations, the complex mixing conductance $G^{\uparrow \downarrow}$ describes the transport of spins noncollinear to the magnetizations of the ferromagnetic reservoirs. When $\operatorname{Im} G^{\uparrow \downarrow} \neq 0$, the total conductance of the system in the presence of a magnetic field can be asymmetric with respect to time reversal. The total conductance changes nonmonotonically with the magnetic field strength for different magnetic configurations. This modulation of the conductance is due to the precession of the spin accumulation in the normal metal. The difference between the conductance of the parallel and antiparallel configurations can be either positive or negative as a function of the applied magnetic field. This effect should be best observable on $\mathrm{Al}$ single crystals attached to ferromagnetic electrodes by means of tunnel junctions or metallic contacts.
\end{abstract}

\section{INTRODUCTION}

In hybrid systems of ferromagnetic and normal metals, interesting phenomena can appear due the interplay between charge and spin. The discovery of the giant magnetoresistance $(\mathrm{GMR})$ effect in metallic magnetic multilayers ${ }^{1}$ has motivated a large number of studies on the transport properties of such systems. ${ }^{2}$ The GMR is caused by spin-dependent scattering in the system. Most studies concentrated on collinear configurations (parallel and antiparallel configurations). There are several papers which cover noncollinear magnetizations, both theoretical ${ }^{3}$ and experimental. ${ }^{4}$

Magnetoelectronic multiterminal devices reveal interesting physics, ${ }^{5}$ but may also lead to different applications, e.g., nonvolatile electronics. Johnson and Silsbee investigated spin-dependent effects in a three-terminal device. ${ }^{5}$ They found transistor effects that depend on the relative orientation of the magnetization of the ferromagnets. ${ }^{6}$ More recently, a ferromagnetic single-electron transistor in a threeterminal configuration has been realized $^{7}$ and studied theoretically. ${ }^{8}$ In this case the source-drain current also depends on the relative orientation of the magnetizations.

Brataas et al. ${ }^{9}$ give a unified semiclassical picture for electron and spin transport in such systems. Their formalism is inspired by the circuit theory of the Andreev reflection, ${ }^{10}$ and is applicable to systems with noncollinear magnetization directions and an arbitrary number and variety of contacts between the ferromagnetic and the normal metals. However, the simple circuit theory of Ref. 9 only holds when the resistances of the contacts between the ferromagnetic and the normal metals are much higher than the resistance of the normal metal itself, thus fails when the size $L$ of the system in the transport direction becomes too large. Moreover, when the size of the system is larger than the spin diffusion length $\left(L \gg l_{s f}\right)$, the presence of spin diffusion in the normal metal requires a more complicated description with spatially de- pendent spin distribution functions.

In the present paper, we present a study of the transport properties of simple ferromagnet/normal-metal/ferromagnet FNF systems (see Fig. 1), taking into account different magnetizations of the ferromagnetic reservoirs and spin diffusion in the normal metal. At low temperatures, spin flip can be due to spin-orbit interactions and scattering by defects or impurities. Exchange scattering by paramagnetic impurities also flips the spin (see, e.g., Appendix A in Ref. 11). The length of the normal metal $L$ is assumed to be much larger than the mean-free path $l_{f}$, so electronic transport may be described by the diffusion equation. On the other hand, we allow the spin diffusion length $l_{s f}$, which is the length scale on which an electron loses its spin in diffusive transport, to be much smaller, of the same order, or much larger than the size of the system $L$. Under an applied bias, ferromagnetic reservoirs inject a spin current, causing a nonequilibrium magnetization or "spin accumulation" in the normal metal. We are interested in the different mechanisms that reduce and also rotate this spin accumulation. For noncollinear configurations the physics of spin injection is more subtle than in the simple collinear case, since it requires generalized

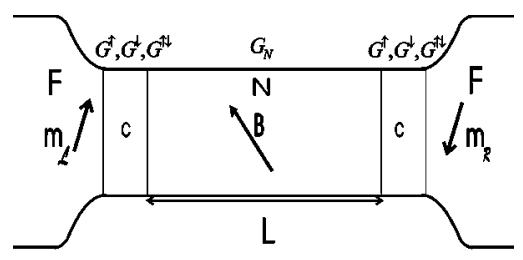

FIG. 1. Ferromagnetic reservoirs attached to diffusive normal metal through arbitrary contacts. Arbitrary but fixed magnetization directions of the ferromagnetic reservoirs and orientations of a magnetic field applied to the normal metal are taken into account. The contacts are described by the spin-dependent conductances $G^{\uparrow}, G^{\downarrow}, G^{\uparrow \downarrow}$ and the normal metal is characterized by the normalmetal conductance $G_{N}$. The ferromagnetic reservoirs are supposed to be large enough and in local equilibrium. 
boundary conditions for transport through a single ferromagnetic normal-metal (FN) contact. ${ }^{9}$ In general, such a contact is charaterized not only by the conventional spin-dependent conductances $G^{\uparrow}, G^{\downarrow}$, which describe the transport of spins collinear to the magnetization of the ferromagnetic reservoir, but also by the (complex) mixingconductance $G^{\uparrow \downarrow}$ (see Ref. 9), that contains information about the transport of spins oriented perpendicular to the magnetization of the ferromagnetic reservoir.

We are also interested in the effect of a magnetic field applied to the diffusive normal metal in arbitrary directions. In this case we assume that the magnetic field only couples to the spin degrees of freedom. Our approach is similar to the treatment of a precessing magnetic field applied to a diffusive metal in Ref. 12.

In Sec. II we introduce and solve the basic equations for the diffusive spin transport, showing the general expression for the nonequilibrium distribution function in the normal metal. In Sec. III we discuss the boundary conditions of the problem. In Sec. IV we obtain analytical expressions for the total conductance of the system in collinear configurations and in the absence of applied magnetic field. We also obtain analytical expressions for the total conductance in the case of noncollinear magnetization directions, zero magnetic field, and no spin-flip scattering. In Sec. V we calculate numerically the conductance in the general case. In Sec. VI we summarize and discuss our results.

\section{DIFFUSIVE SPIN TRANSPORT}

When a bias is applied to our FNF device, a spin current is injected from the ferromagnetic reservoirs into the normal metal, causing a nonequilibrium magnetization or spin accumulation. For an arbitrary magnetic configuration of the system, the spin accumulated in the normal metal can be oriented in differents directions. If we take the spin quantization axis parallel to the magnetization of one of the ferromagnetic reservoirs, we need to take into account spins oriented perpendicular to this quantization axis, which can be described as a superposition of up $(\uparrow)$ and down $(\downarrow)$ spin states. We study a geometry invariant to translations in the lateral direction, so all quantities depend only on one spatial coordinate $(x)$. The spin-polarized electron distribution is characterized by a $2 \times 2$ matrix in spin space of the form

$$
\hat{f}^{N}(x)=\left(\begin{array}{ll}
f_{\uparrow \uparrow}^{N}(x) & f_{\uparrow \downarrow}^{N}(x) \\
f_{\downarrow \uparrow}^{N}(x) & f_{\downarrow \downarrow}^{N}(x)
\end{array}\right) .
$$

When the size of the system $L$ is larger than the spin diffusion length $l_{s f}, \hat{f}^{N}(x)$ depends on the position. Here we are interested in transport under the condition $l_{f} \ll l_{s f}$, where $l_{f}=v_{F}\left(1 / \tau+1 / \tau_{s f}\right)^{-1}$ is the mean free path, $v_{F}$ is the Fermi velocity, $\tau$ the spin-conserving scattering time and $\tau_{s f}$ the spin-flip scattering time. Both $\tau$ and $\tau_{s f}$ are considered isotropic in momentum space. The spin diffusion length $l_{s f}$ is defined as $l_{s f}=\sqrt{D \tau_{s f}}$, where $D=v_{F} l_{f} / d$, is the spinindependent diffusion coefficient of the normal metal $(d$ $=1,2,3$ is the dimension of the normal metal). So under the condition $l_{f} \ll l_{s f}$, we obtain for diffusive spin transport in the steady state the following $2 \times 2$ matrix equations for $\hat{f}^{N}(x)$ :

$$
\begin{gathered}
D \frac{\partial^{2} \hat{f}^{N}(x)}{\partial x^{2}}=\frac{1}{\tau_{s f}}\left(\hat{f}^{N}(x)-\hat{\mathbf{1}} \frac{\operatorname{Tr}\left[\hat{f}^{N}(x)\right]}{2}\right) \\
\hat{J}^{N}(x)=-D \frac{\partial \hat{f}^{N}(x)}{\partial x},
\end{gathered}
$$

where $\hat{\mathbf{1}}$ is the unit matrix and where the electron charge $e$ is assumed to be equal to one. Equation (2) describes the relaxation of the spin accumulation due to spin-flip scattering, and Eq. (3) relates the current density matrix $\hat{j}^{N}(x)$ and $\hat{f}^{N}(x)$. In the case of collinear transport, our matrix equations simply reduce to

$$
\begin{gathered}
\frac{\partial^{2} f_{s}^{N}(x)}{\partial x^{2}}=\frac{1}{2} \frac{f_{s}^{N}(x)-f_{-s}^{N}(x)}{l_{s f}^{2}}, \\
j_{s}^{N}(x)=-D \frac{\partial f_{s}^{N}(x)}{\partial x},
\end{gathered}
$$

where $s=(\uparrow, \downarrow)$. Equations (4) and (5) have been extensively used for collinear transport in FN multilayers in which the current flows perpendicular to the planes of the interfaces (CPP geometry). ${ }^{11,13}$

We are also interested in the effect of an external magnetic field applied to the normal metal in an arbitrary direction. We know that the magnetic Zeeman energy associated with the coupling between the magnetic field and the spin of the electrons is given by $g \mu_{B} \hat{\sigma} \cdot \vec{B} / 2$, where $\mu_{B}$ is the Bohr magneton, $g$ is the gyromagnetic ratio, $\hat{\sigma}=\left(\hat{\sigma}_{x}, \hat{\sigma}_{y}, \hat{\sigma}_{z}\right)$ is the vector of Pauli matrices and $\vec{B}$ is the external magnetic field. Semiclassically, we can write for the spin dynamics (see, e.g., Ref. 14)

$$
\frac{\partial \hat{f}^{N}(x)}{\partial t}=\frac{i}{\hbar}\left[\frac{g \mu_{B}}{2}(\hat{\sigma} \cdot \vec{B}), \hat{f}^{N}(x)\right]_{-} .
$$

Then, in the steady state

$$
\begin{aligned}
D \frac{\partial^{2} \hat{f}^{N}(x)}{\partial x^{2}}= & \frac{1}{\tau_{s f}}\left(\hat{f}^{N}(x)-\hat{\mathbf{1}} \frac{\operatorname{Tr}\left[\hat{f}^{N}(x)\right]}{2}\right) \\
& -\frac{i}{\hbar}\left[\frac{g \mu_{B}}{2}(\hat{\sigma} \cdot \vec{B}), \hat{f}^{N}(x)\right]_{-} .
\end{aligned}
$$

Using the properties of the Pauli matrices we can express the nonequilibrium distribution matrix $\hat{f}^{N}(x)$ as

$$
\hat{f}^{N}(x)=f_{0}(x) \hat{\mathbf{1}}+\hat{\boldsymbol{\sigma}} \cdot \vec{f}(x),
$$

where $f_{0}(x)$ is a scalar and $\vec{f}(x)=\left[f_{x}(x), f_{y}(x), f_{z}(x)\right]$ is a three component vector. $f_{0}(x)$ is the particle or spinindependent distribution function. On the other hand, $f_{z}(x)$ describes the "spin polarization" on the system, and $f_{x}(x)$ and $f_{y}(x)$ contain information about the spins oriented perpendicular to the quantization axis. We call the three com- 
ponent vector $\vec{f}(x)$ the spin-dependent distribution function. Using Eq. (8), we separate Eq. (7) into two contributions, one for the spin-independent part and another for the spindependent part:

$$
\begin{gathered}
\frac{\partial^{2} f_{0}(x)}{\partial x^{2}}=0, \\
\frac{\partial^{2} \vec{f}(x)}{\partial x^{2}}=\frac{1}{l_{s f}^{2}} \vec{f}(x)+\left(\frac{g \mu_{B}}{\hbar} \frac{\vec{B}}{D} \times \vec{f}(x)\right) .
\end{gathered}
$$

The spin-independent part [Eq. (9a)], is the conventional result for diffusive particle transport. Similar to Eq. (5), the particle current density $j_{0}^{N}(x)$ reads

$$
j_{0}^{N}(x)=-D \frac{\partial\left\{\operatorname{Tr}\left[\hat{f}^{N}(x)\right]\right\}}{\partial x}=-2 D \frac{\partial f_{0}(x)}{\partial x} .
$$

Equations (9a) and (10) express the particle current conservation

$$
\frac{\partial j_{0}^{N}(x)}{\partial x}=0
$$

The general solution of Eq. (9a) is

$$
f_{0}(x)=\mathcal{P}+\mathcal{O} x .
$$

Equation (9b) describes how the spin accumulation relaxes by spin-flip scattering and by the spin precession around the magnetic field. This equation can be written in a general matrix form as

$$
\frac{\partial^{2} \vec{f}(x)}{\partial x^{2}}=\mathbf{A} \vec{f}(x) .
$$

The eigenvalues associated with the matrix $\mathbf{A}$ are

$$
\begin{gathered}
\lambda_{o}=\frac{1}{l_{s f}^{2},} \\
\lambda_{+}=\frac{1}{l_{s f}^{2}}+i|\vec{h}|, \\
\lambda_{-}=\frac{1}{l_{s f}^{2}}-i|\vec{h}|,
\end{gathered}
$$

where we have introduced the vector $\vec{h}=g \mu_{B} \vec{B} / \hbar D$, which describes the "effectiveness" of the magnetic field in a diffusive metal. The eigenvector associated with $\lambda_{o}$ is

$$
\vec{v}_{o}=\frac{1}{|\vec{h}|}\left(\begin{array}{c}
h_{x} \\
h_{y} \\
h_{z}
\end{array}\right) .
$$

On the other hand, $\lambda_{+}$and $\lambda_{-}$have associated two complex conjugated eigenvectors $\vec{v}_{+}=\vec{v}_{1}+i \vec{v}_{2}$ and $\vec{v}_{-}=\vec{v}_{1}-i \vec{v}_{2}$, where

$$
\vec{v}_{1}=\frac{1}{\sqrt{\left(h_{x}^{2}+h_{y}^{2}\right)}|\vec{h}|}\left(\begin{array}{c}
h_{x} h_{z} \\
h_{y} h_{z} \\
-\left(h_{z}^{2}+h_{x}^{2}\right)
\end{array}\right)
$$

and

$$
\vec{v}_{2}=\frac{1}{\sqrt{h_{x}^{2}+h_{y}^{2}}}\left(\begin{array}{c}
h_{y} \\
-h_{x} \\
0
\end{array}\right) \text {. }
$$

The general solution of Eq. (9b), can then be written in terms of the eigenvalues $\lambda_{o}, \lambda_{+}, \lambda_{-}$and vectors $\vec{v}_{o}, \vec{v}_{1}, \vec{v}_{2}$ as

$$
\vec{f}(x)=\left\{\begin{array}{l}
\mathcal{A} \vec{v}_{o} \cosh \left(x / l_{s f}\right)+\mathcal{B} \vec{v}_{o} \sinh \left(x / l_{s f}\right) \\
+\mathcal{C}\left[\vec{v}_{1} \cosh (X) \cos (Y)-\vec{v}_{2} \sinh (X) \sin (Y)\right] \\
-\mathcal{D}\left[\vec{v}_{1} \sinh (X) \sin (Y)+\vec{v}_{2} \cosh (X) \cos (Y)\right] \\
+\mathcal{E}\left[\vec{v}_{1} \sinh (X) \cos (Y)-\vec{v}_{2} \cosh (X) \sin (Y)\right] \\
-\mathcal{F}\left[\vec{v}_{1} \cosh (X) \sin (Y)+\vec{v}_{2} \sinh (X) \cos (Y)\right] .
\end{array}\right\}
$$

where

$$
\begin{gathered}
X=\sqrt{\frac{1+\sqrt{1+\alpha^{2}}}{2} \frac{x}{l_{s f}}} \\
Y=\sqrt{\frac{-1+\sqrt{1+\alpha^{2}}}{2}} \frac{x}{l_{s f}} .
\end{gathered}
$$

and where the dimensionless constant $\alpha=g \omega_{L} \tau_{s f}=|\vec{h}| l_{s f}^{2}$ is the ratio between spin-flip and precession relaxation mechanisms. $\omega_{L}=\mu_{B}|\vec{B}| / \hbar$ is the (Larmor) frequency for the spin precession around the magnetic field. ${ }^{15}$ The solution associated with $\lambda_{o}$ describes the relaxation of the spin accumulation due to spin-flip scattering, and the two complex conjugated solutions associated with $\lambda_{+}$and $\lambda_{-}$describe the relaxation and precession of the spins due to the coupling with the magnetic field. The eight real constants $(\mathcal{O}, \mathcal{P}, \mathcal{A}, \mathcal{B}, \mathcal{C}, \mathcal{D}, \mathcal{E}, \mathcal{F})$ must be determined by the boundary conditions.

\section{BOUNDARY CONDITIONS}

We consider two ferromagnetic reservoirs attached to a diffusive normal metal through some arbitrary contacts, as shown in Fig. 1. The ferromagnetic reservoirs are supposed to be large and in local equilibrium at chemical potentials $\mu_{\mathcal{L}, \mathcal{R}}(\mathcal{L}, \mathcal{R}$ denotes left and right reservoir, respectively), and with energy-dependent diagonal distribution matrices in spin space $\hat{f}_{\mathcal{L}, \mathcal{R}}^{F}(\epsilon)$. The components of $\hat{f}_{\mathcal{L}, \mathcal{R}}^{F}(\epsilon)$ are given by the Fermi-Dirac distribution function $f^{F D}\left(\epsilon, \mu_{\mathcal{L}, \mathcal{R}}\right)$, and the direction of the magnetization in each ferromagnetic reservoir is denoted by the unit vector $\overrightarrow{\mathbf{m}}_{\mathcal{L}, \mathcal{R}}$. The current through the system and the nonequilibrium distribution function in the normal metal are completely determined by the relative orientation of the magnetization directions in the ferromagnetic reservoirs, the contact conductances, the normal-metal 


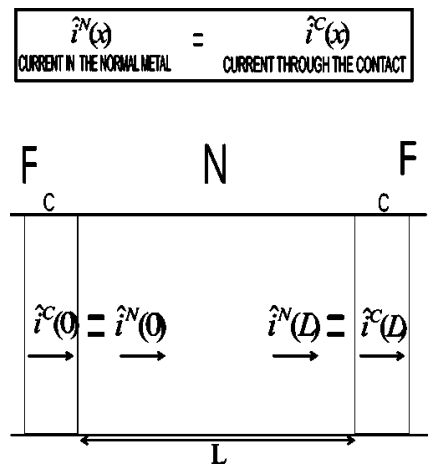

FIG. 2. Current conservation imposes boundary conditions on the system. The current through the contacts $\hat{\imath}^{C}(x)$ is equal to the current into the normal metal $\hat{\imath}^{N}(x)$ at each contact. $\hat{\imath}^{C}(x)$ depends on the contact conductances and on the direction of the magnetization of the adjacent ferromagnet reservoir and $\hat{\imath}^{N}(x)$ is the current for the normal metal.

conductance, the spin-diffusion length, and the magnetic field.

The current through an FN contact is given in Ref. 9 in terms of the microscopic scattering matrices of the LandauerBüttiker formalism. ${ }^{16}$ According to Eq. (3) of Ref. 9, the particle current through a single contact directed into the normal metal can be written as

$$
\begin{aligned}
\hat{i}^{C}(x)= & G^{\uparrow} \hat{u}^{\uparrow}\left[\hat{f}^{F}-\hat{f}^{N}(x)\right] \hat{u}^{\uparrow}+G^{\downarrow} \hat{u}^{\downarrow}\left[\hat{f}^{F}-\hat{f}^{N}(x)\right] \hat{u}^{\downarrow} \\
& -G^{\uparrow \downarrow} \hat{u}^{\uparrow} \hat{f}^{N}(x) \hat{u}^{\downarrow}-\left(G^{\uparrow \downarrow}\right)^{*} \hat{u}^{\downarrow} \hat{f}^{N}(x) \hat{u}^{\uparrow},
\end{aligned}
$$

where $\hat{f}^{N}(x)$ and $\hat{f}_{\mathcal{L}, \mathcal{R}}^{F}$ are isotropic distribution functions, $G^{\uparrow}$ and $G^{\downarrow}$ are the conventional spin-dependent conductances, which describe the transport of spins oriented in the direction of the magnetization of the adjacent ferromagnetic reservoir, and $G^{\uparrow \downarrow}=\operatorname{Re} G^{\uparrow \downarrow}+i \operatorname{Im} G^{\uparrow \downarrow}$ is the mixing conductance, which contains information about the transport of spins oriented in perpendicular direction to the magnetization of the ferromagnetic reservoir. The matrices $\hat{u}^{\uparrow}=(\hat{\mathbf{1}}+\hat{\boldsymbol{\sigma}} \cdot \overrightarrow{\mathbf{m}}) / 2$, and $\hat{u}^{\downarrow}=(\hat{1}-\hat{\sigma} \cdot \overrightarrow{\mathbf{m}}) / 2$ define the basis in which the spinquantization axis is parallel to the magnetization of the ferromagnet (for details, see Ref. 17). Equation (13) relates the spin current through the contact $\hat{l}^{C}(x)$ and the nonequilibrium distribution matrix $\hat{f}^{N}(x)$ in the normal metal. Due to current conservation, Eq. (13) is equal, at each contact, to the particle current per energy interval in the normal metal (see Fig. 2). The particle current per energy interval is related with the current density $\hat{j}^{N}(x)$ as, $\hat{i}^{N}(x)=S \nu_{D O S} \hat{j}^{N}(x)$, where $S$ is the surface perpendicular to the transport direction and $\nu_{D O S}$ is the density of states of the normal metal. Using Eq. (3) $\hat{i}^{N}(x)$ is

$$
\hat{i}^{N}(x)=-S \nu_{D O S} D \frac{\partial \hat{f}^{N}(x)}{\partial x} .
$$

So we have

$$
\hat{i}^{C}\left(x=0^{+}\right)=\hat{i}^{N}\left(x=0^{+}\right)
$$

for the left contact $\left(x=0^{+}\right)$and

$$
\hat{i}^{N}\left(x=L^{-}\right)=\hat{\imath}^{C}\left(x=L^{-}\right)
$$

for the right contact $\left(x=L^{-}\right)$. By substituting Eqs. (13) and (14) into Eqs. (15a) and (15b), we obtain the boundary conditions for the left contact $\left(x=0^{+}\right)$:

$$
\begin{aligned}
- & S \nu_{D O S} D{\frac{\partial \hat{f}^{N}(x)}{\partial x}}_{x=0^{+}}+G^{\uparrow} \hat{u}^{\uparrow} \hat{f}^{N}\left(0^{+}\right) \hat{u}^{\uparrow}+G^{\downarrow} \hat{u}^{\downarrow} \hat{f}^{N}\left(0^{+}\right) \hat{u}^{\downarrow} \\
& +G^{\uparrow \downarrow} \hat{u}^{\uparrow} \hat{f}^{N}\left(0^{+}\right) \hat{u}^{\downarrow}+\left(G^{\uparrow \downarrow}\right)^{*} \hat{u}^{\downarrow} \hat{f}^{N}\left(0^{+}\right) \hat{u}^{\uparrow} \\
= & G^{\uparrow} \hat{u}^{\uparrow} \hat{f}_{\mathcal{L}^{F}}^{F} \hat{u}^{\uparrow}+G^{\downarrow} \hat{u}^{\downarrow} \hat{f}_{\mathcal{L}^{F}}^{F} \hat{u}^{\downarrow},
\end{aligned}
$$

and for the right contact $\left(x=L^{-}\right)$:

$$
\begin{aligned}
& S \nu_{D O S} D{\frac{\partial \hat{f}^{N}(x)}{\partial x}}_{x=L^{-}}+G^{\uparrow} \hat{u}^{\uparrow} \hat{f}^{N}\left(L^{-}\right) \hat{u}^{\uparrow}+G^{\downarrow} \hat{u}^{\downarrow} \hat{f}^{N}\left(L^{-}\right) \hat{u}^{\downarrow} \\
& +G^{\uparrow \downarrow} \hat{u}^{\uparrow} \hat{f}^{N}\left(L^{-}\right) \hat{u}^{\downarrow}+\left(G^{\uparrow \downarrow}\right)^{*} \hat{u}^{\downarrow} \hat{f}^{N}\left(L^{-}\right) \hat{u}^{\uparrow} \\
& =G^{\uparrow} \hat{u}^{\uparrow} \hat{f}_{\mathcal{R}}^{F} \hat{u}^{\uparrow}+G^{\downarrow} \hat{u}^{\downarrow} \hat{f}_{\mathcal{R}}^{F} \hat{u}^{\downarrow} .
\end{aligned}
$$

The set of parameters $\left\{G^{\uparrow}, G^{\downarrow}, \operatorname{Re} G^{\uparrow \downarrow}, \operatorname{Im} G^{\uparrow \downarrow}, \hat{u}^{\uparrow}, \hat{u}^{\downarrow}\right\}$ is in general different for each contact, but we have omitted the indices $\mathcal{L}$ and $\mathcal{R}$ in Eqs. (16a) and (16b) for brevity. Equations (16a) and (16b) are two $2 \times 2$ matrix equations, that provide us a system of linear equations that determinate the eight unknown constants $(\mathcal{O}, \mathcal{P}, \mathcal{A}, \mathcal{B}, \mathcal{C}, \mathcal{D}, \mathcal{E}, \mathcal{F})$.

From Eq. (10) we can see that the total particle current $i_{0}^{N}$ can be written in terms of one of these constants as

$$
i_{0}^{N}=-2 S \nu_{D O S} D \frac{\partial f_{0}(x)}{\partial x}=-2 D S \nu_{D O S} \mathcal{O}=-2 V_{o l} G_{N} \mathcal{O},
$$

where $G_{N}=(D / L) \nu_{D O S}$ is the normal-metal conductance and $V_{o l}$ is the volume of the normal metal. By solving the system of Eqs. (16a) and (16b), we can calculate this total particle current. $i_{0}^{N}$ is proportional to the difference between the distribution functions of the ferromagnets $i_{0}^{N} \propto\left(f_{\mathcal{L}}^{F}-f_{\mathcal{R}}^{F}\right)$, times a quantity which does not depends on energy. From this quantity it is possible to obtain the total conductance $G^{T}$,

$$
i_{0}^{N}=G^{T}\left(f_{\mathcal{L}}^{F}-f_{\mathcal{R}}^{F}\right),
$$

where $G^{T}$ is in principle a function of the relative orientation of the magnetization directions in the ferromagnetic reservoirs, the contacts and normal-metal conductances, the spindiffusion length, and also of the magnetic field: ${ }^{18}$

$$
G^{T} \equiv G^{T}\left(\overrightarrow{\mathbf{m}}_{\mathcal{L}, \mathcal{R}},\left\{G^{\uparrow}, G^{\downarrow}, \operatorname{Re} G^{\uparrow \downarrow} \operatorname{Im} G^{\uparrow \downarrow}\right\}_{\mathcal{L}, \mathcal{R}}, G_{N}, l_{s f}, \vec{B}\right) .
$$

By studying $G^{T}$ for different values of these parameters, we obtain information about the physics of the spin accumulation in diffusive systems.

\section{ANALYTICAL EXPRESSIONS}

The properties of the contacts are parametrized by the spin-dependent conductances $\quad\left\{G^{\uparrow}, G^{\downarrow}, \operatorname{Re} G^{\uparrow \downarrow} \operatorname{Im} G^{\uparrow \downarrow}\right\}_{\mathcal{L}, \mathcal{R}}$. For collinear configurations of the ferromagnetic reservoirs (parallel and antiparallel), it is easy to obtain simple expressions for the conductance, which can be interpreted by simple equivalent circuits. When $l_{s f} \gg L$, there is no mixing 
between spin-up $(\uparrow)$ and spin-down $(\downarrow)$ channels, and we obtain the conductance for the parallel configuration,

$$
G_{P}=\frac{G_{\mathcal{L}}^{\uparrow} G_{\mathcal{R}}^{\uparrow} G_{N}}{\left(G_{\mathcal{L}}^{\uparrow}+G_{\mathcal{R}}^{\uparrow}\right) G_{N}+G_{\mathcal{L}}^{\uparrow} G_{\mathcal{R}}^{\uparrow}}+\frac{G_{\mathcal{L}}^{\downarrow} G_{\mathcal{R}}^{\downarrow} G_{N}}{\left(G_{\mathcal{L}}^{\downarrow}+G_{\mathcal{R}}^{\downarrow}\right) G_{N}+G_{\mathcal{L}}^{\downarrow} G_{\mathcal{R}}^{\downarrow}}
$$

and for the antiparallel configuration,

$$
G_{A P}=\frac{G_{\mathcal{L}}^{\uparrow} G_{\mathcal{R}}^{\downarrow} G_{N}}{\left(G_{\mathcal{L}}^{\uparrow}+G_{\mathcal{R}}^{\downarrow}\right) G_{N}+G_{\mathcal{L}}^{\uparrow} G_{\mathcal{R}}^{\downarrow}}+\frac{G_{\mathcal{L}}^{\downarrow} G_{\mathcal{R}}^{\uparrow} G_{N}}{\left(G_{\mathcal{L}}^{\downarrow}+G_{\mathcal{R}}^{\uparrow}\right) G_{N}+G_{\mathcal{L}}^{\downarrow} G_{\mathcal{R}}^{\uparrow}} .
$$

On the other hand, when $l_{s f} \ll L$, spin-up ( $\left.\uparrow\right)$ and spin-down $(\downarrow)$ channels are completely mixed due to spin-flip scattering and the spin accumulation vanishes. In this limit we have

$$
G^{0}=\left(\frac{1}{2 G_{N}}+\frac{1}{G_{\mathcal{L}}^{\uparrow}+G_{\mathcal{L}}^{\downarrow}}+\frac{1}{G_{\mathcal{R}}^{\uparrow}+G_{\mathcal{R}}^{\downarrow}}\right)^{-1} .
$$

These expressions correspond to the simple equivalent circuits displayed in Fig. 3. Equation (18a) corresponds to a circuit in which the two spin channels are independent in the parallel configuration [Fig. 3(a)]. Equation (18b) corresponds to the antiparallel configuration [Fig. 3(b)]. Equation (19) is equivalent to a circuit with a complete mixing between spin up $(\uparrow)$ and spin-down $(\downarrow)$ channels [Fig. 3(c)], in which there is no difference between parallel and antiparallel configurations.

For symmetric contacts $\quad\left(G_{\mathcal{L}}^{\uparrow}=G_{\mathcal{R}}^{\uparrow}=G^{\uparrow}\right.$ and $G_{\mathcal{L}}^{\downarrow}=G_{\mathcal{R}}^{\downarrow}$ $=G^{\downarrow}$ ), we find analytical expressions for the conductance of the system for any value of $L / l_{s f}$, in the parallel configuration,

$$
G_{P}^{S}=2 G_{N} \frac{2 G^{\uparrow} G^{\downarrow} \frac{l_{s f}}{L} \tanh \left(\frac{L}{2 l_{s f}}\right)+G G_{N}}{G_{N}\left(4 G_{N}+G\right)+2\left(G_{N} G+G^{\uparrow} G^{\downarrow}\right) \frac{l_{s f}}{L} \tanh \left(\frac{L}{2 l_{s f}}\right)},
$$

and in the antiparallel configuration,

$$
G_{A P}^{S}=2 G_{N} \frac{2 G^{\uparrow} G^{\downarrow} \frac{l_{s f}}{L}+G G_{N} \tanh \left(\frac{L}{2 l_{s f}}\right)}{G_{N}\left(4 G_{N}+G\right) \tanh \left(\frac{L}{2 l_{s f}}\right)+2\left(G_{N} G+G^{\uparrow} G^{\downarrow}\right) \frac{l_{s f}}{L}},
$$

where $G=G^{\uparrow}+G^{\downarrow}$.

In the limit $l_{s f} \gg L$, these equations reduce to, for parallel configuration,
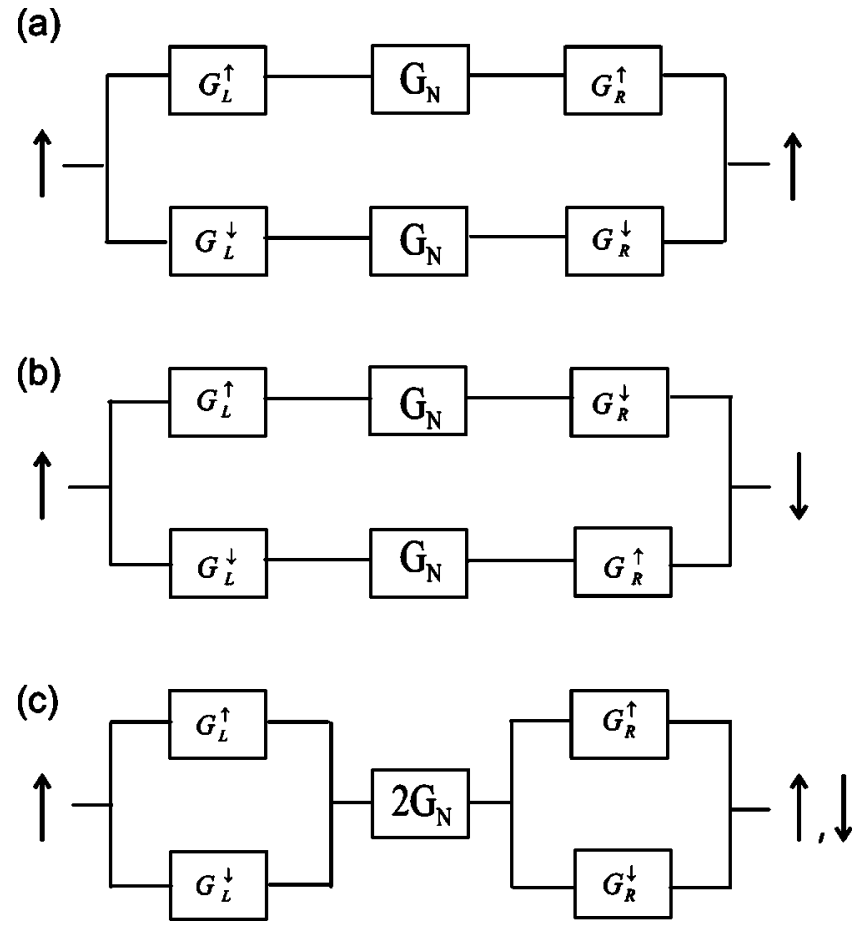

FIG. 3. Equivalent circuits for parallel and antiparallel configurations in the limits $l_{s f} \gg L$ and $l_{s f} \ll L$. (a) and (b) correspond to the parallel and antiparallel configuration, respectively, when $l_{s f} \gg L$. In this limit, the two spin channels are independent and there is no mixing between them; (c) parallel and antiparallel configurations when $l_{s f} \ll L$. In this case, there is complete mixing between spin-up $(\uparrow)$ and spin-down $(\downarrow)$ channels and the spin accumulation vanishes.

$$
G_{P}^{S}=\frac{G^{\uparrow} G_{N}}{G^{\uparrow}+2 G_{N}}+\frac{G^{\downarrow} G_{N}}{G^{\downarrow}+2 G_{N}},
$$

for antiparallel configuration,

$$
G_{A P}^{S}=2 \frac{G^{\uparrow} G^{\downarrow} G_{N}}{G G_{N}+G^{\uparrow} G^{\downarrow}},
$$

and in the limit $\left(l_{s f} \ll L\right)$ for parallel and antiparallel configurations,

$$
G^{0}=\frac{G G_{N}}{2 G_{N}+G / 2}
$$

For noncollinear configurations there is no simple circuit analogy, but we can still find an analytical expression for the total conductance of the system as a function of the angle between the magnetizations of the different ferromagnets $\theta$, when $l_{s f} \gg L$, at zero magnetic field $(\vec{B}=0)$ and for symmetric contacts:

$$
G^{T}(\theta)=2 G_{N} \times\left(1-\frac{\left|G^{\uparrow \downarrow}\right|^{2}\left[4 G_{N}^{2}(1+\cos \theta)+2 G G_{N}\right]+2 \operatorname{Re} G^{\uparrow \downarrow} G_{N}^{2} G(1-\cos \theta)}{\left|G^{\uparrow \downarrow}\right|^{2}\left[\left(4 G_{N}^{2}+G G_{N}\right)(1+\cos \theta)+2\left(G G_{N}+G^{\uparrow} G^{\downarrow}\right)\right]+2 \operatorname{Re} G^{\uparrow \downarrow} G_{N}\left[\left(G G_{N}+G^{\uparrow} G^{\downarrow}\right)(1-\cos \theta)\right]}\right)
$$


In the limit of $\theta=0$ and $\theta=\pi$, Eq. (23) simplifies to Eqs. (21a) and (21b), respectively. When the resistance of the normal metal is negligible compared to the contacts resistance $\left(G_{N} \rightarrow \infty\right)$, this reduces to

$$
G^{T}(\theta)=\frac{G}{2}\left(1-p^{2} \frac{\tan ^{2} \theta / 2}{\tan ^{2} \theta / 2+|\eta|^{2} / \operatorname{Re}(\eta)}\right),
$$

where $p=P / G=\left(G^{\uparrow}-G^{\downarrow}\right) / G$ is the polarization and $\eta$ $=2 G^{\uparrow \downarrow} / G$ is the (complex) relative mixing conductance. Equation (24) can also be obtained by means of the circuit theory. ${ }^{10,17}$

\section{NUMERICAL RESULTS}

The total conductance depends on the spin-dependent conductances of the contacts. We mostly set the polarization $p=P / G=\left(G^{\uparrow}-G^{\downarrow}\right) / G=0.5$ (for real metallic ferromagnets like $\mathrm{Fe}$ or $\mathrm{Co}, p$ is 0.4 and 0.35 , respectively ${ }^{19}$ ), which corresponds to a ratio $G^{\uparrow} / G^{\downarrow}=3$. On the other hand, the real part of the mixing conductance obeys $\operatorname{Re} G^{\uparrow \downarrow} \geqslant\left(G^{\uparrow}+\right.$ $\left.G^{\downarrow}\right) / 2 .{ }^{9}$ The conductances of the contacts and the diffusive normal metal are considered to be of the same order $G_{N}$ $\sim\left(G^{\uparrow}, G^{\downarrow}, G^{\uparrow \downarrow}\right)$.

\section{A. Collinear and noncollinear configurations}

The total conductance depends on the magnetic configuration. We plot in Figs. 4(a) and (b), $G^{T} / G_{N}$ as function of the relative angle between magnetizations $\theta$, for symmetric contacts, zero magnetic field $(\vec{B}=0)$ and in the absence of spin relaxation in the normal metal $\left(l_{s f} \gg L\right)$, as given by Eq. (23) for different values of $\operatorname{Im} G^{\uparrow \downarrow}$ and $\operatorname{Re} G^{\uparrow \downarrow}$ respectively. For $\theta=0^{\circ}, \theta=360^{\circ}$, and $\theta=180^{\circ}$ the total conductance does not depend on the mixing conductance and the values of $G^{T} / G_{N}$ at $\theta=0^{\circ}, 360^{\circ}$, and $\theta=180^{\circ}$ are given by Eqs. (21a) and (21b), respectively. On the other hand, for noncollinear configurations, the total conductance increases with increasing mixing conductance (the dip become more sharp). This enhancement is due to the contributions of noncollinear spins to the transport, in which electrons with spins oriented in different directions than the magnetization of the adjacent ferromagnet are transmitted or reflected at the contact. These processes are described by the real and the imaginary part of the mixing conductance.

\section{B. Spin-flip scattering, spin relaxation}

When spin-flip scattering is caused by spin-orbit interaction in the normal metal, the spin-diffusion length $l_{s f}$ can be estimated to be equal to $l_{f} /(\alpha Z)^{2}$, where $\alpha$ is the relativistic fine structure constant, $Z$ is the atomic number, and $l_{f}$ is the mean free path (see, e.g., Ref. 20). In $\mathrm{Co} / \mathrm{Cu}$ multilayers, the spin diffusion length $l_{s f}$ is of the order of a few hundred angstrom (see Appendix A in Ref. 11). For Al, $l_{s f}$ can be estimated to be of the order of a few micrometers for polycrystalline $\mathrm{Al}$ (see Ref. 19), or even between 10 and $70 \mu \mathrm{m}$ for Al single crystals. ${ }^{5}$ In the case of very pure $\mathrm{Na}, \tau_{s f}$ $\sim 1 \mu \mathrm{s.}{ }^{21}$ In this case, $l_{s f}$ limited by spin-orbit interactions can be estimated to be of the order of $0.4 \mathrm{~cm}$. In Fig. 5(a) we plot, at zero magnetic field and for symmetric contacts, the conductance of the system $G^{T}$, normalized to the conduc-

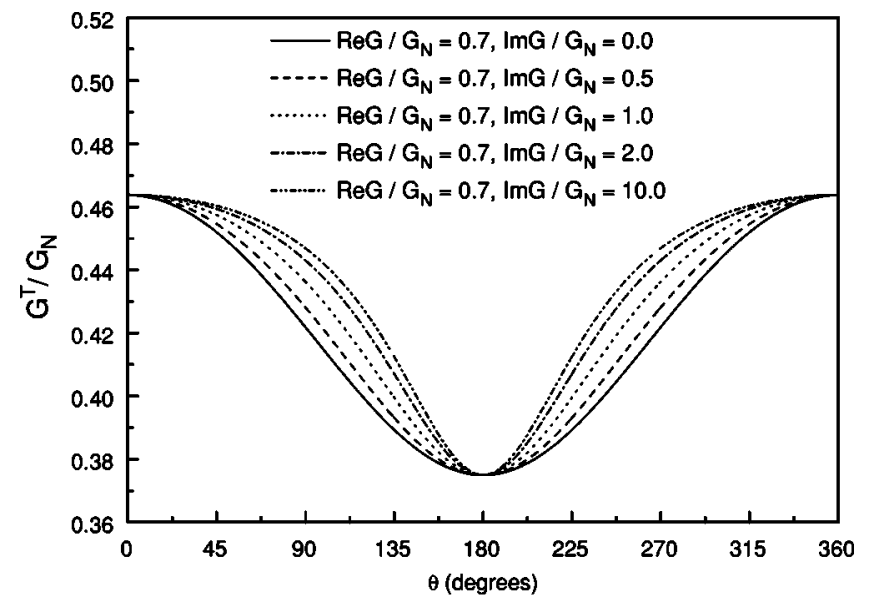

(a)

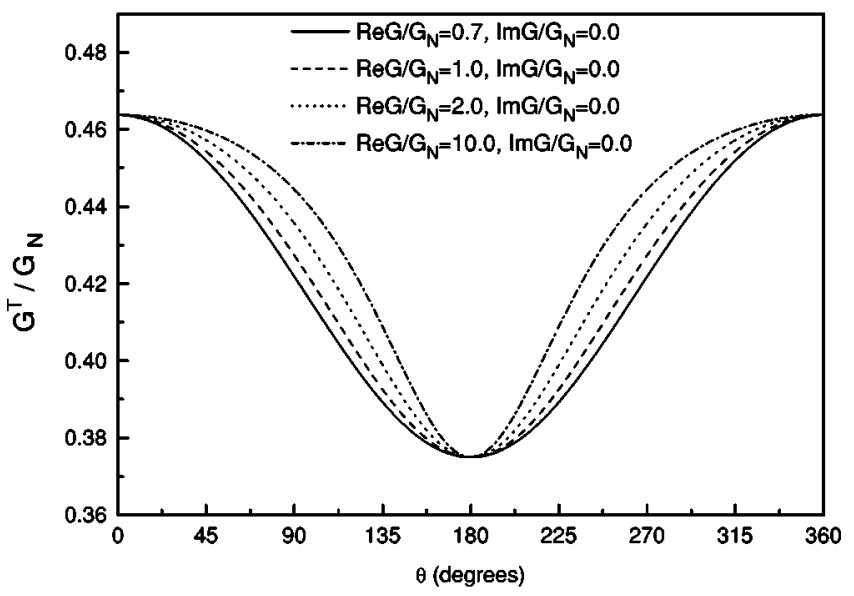

(b)

FIG. 4. Mixing conductance: Dependence of $G^{T} / G_{N}$ on the relative angle $\theta$ between the magnetizations of the ferromagnetic reservoirs, for symmetric contacts, zero magnetic field, and in the absence of spin-flip scattering. (a) The following set of parameters is chosen: $G^{\uparrow} / G_{N}=1.0, G^{\downarrow} / G_{N}=0.3, \operatorname{Re} G^{\uparrow \downarrow} / G_{N}=0.7$, and $\operatorname{Im} G^{\uparrow \downarrow} / G_{N}$ takes values $0.0,0.5,1.0,2.0$, and 10.0 corresponding to the different plotted lines. (b) In this case, $G^{\uparrow} / G_{N}=1.0, G^{\downarrow} / G_{N}$ $=0.3, \operatorname{Im} G^{\uparrow \downarrow} / G_{N}=0.0$, and $\operatorname{Re} G^{\uparrow \downarrow} / G_{N}$ changes with values 0.7 , 1.0, 2.0, and 10.0. According to the condition $\operatorname{Re} G^{\uparrow \downarrow} \geqslant\left(G^{\uparrow}\right.$ $\left.+G^{\downarrow}\right) / 2, \operatorname{Re} G^{\uparrow \downarrow} / G_{N}$ cannot be smaller than 0.65.

tance $G^{0}$ given by Eq. (22), as a function of $L / l_{s f}$. The length of the normal-metal section $L$ is set to be constant, in order to keep a constant value of $G_{N}$. When $l_{s f} \gg L$ the conductance of the system depends on the magnetic configuration. By decreasing $l_{s f}$, all configurations converge to the same value of conductance $G^{T} / G^{0}=1$. All configurations reach the same value of the conductance long before $G^{T} / G^{0}=1$, since for $l_{s f}<L$ both contacts become independent and as the relative magnetic configuration is irrelevant. In Fig. 5(b) we plot $G^{T} / G^{0}$ in the case of antiparallel configuration for differents values of the relative polarization $P / G_{N}=\left(G^{\uparrow}-G^{\downarrow}\right) / G_{N}$ and for $G / G_{N}=\left(G^{\uparrow}+G^{\downarrow}\right) / G_{N}$ constant. When $l_{s f} \gg L$ the configuration with large relative polarization $P / G_{N}$ gives a small conductance and vice versa. The spin accumulation increases with increasing polarization of the ferromagnet and causes a reduction of the total conductance of the system. For $l_{s f} \ll L$ we also see that in each 


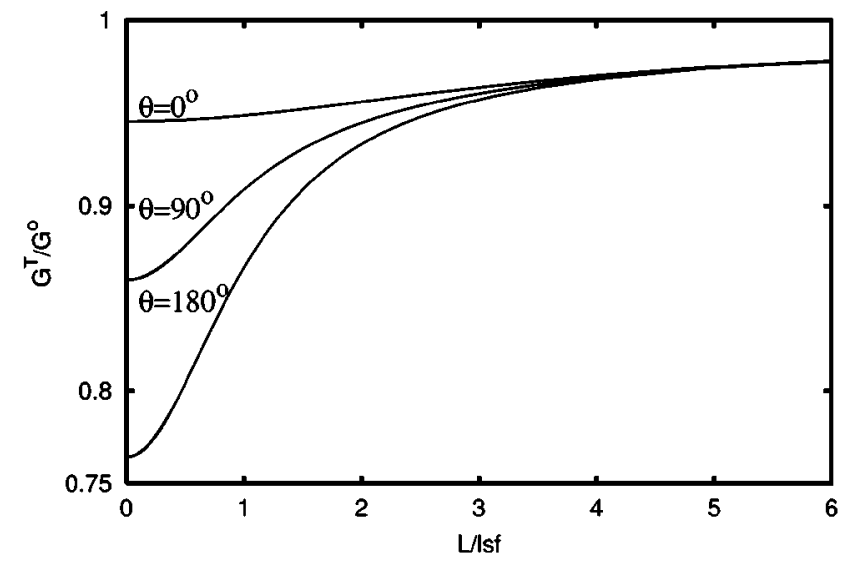

(a)

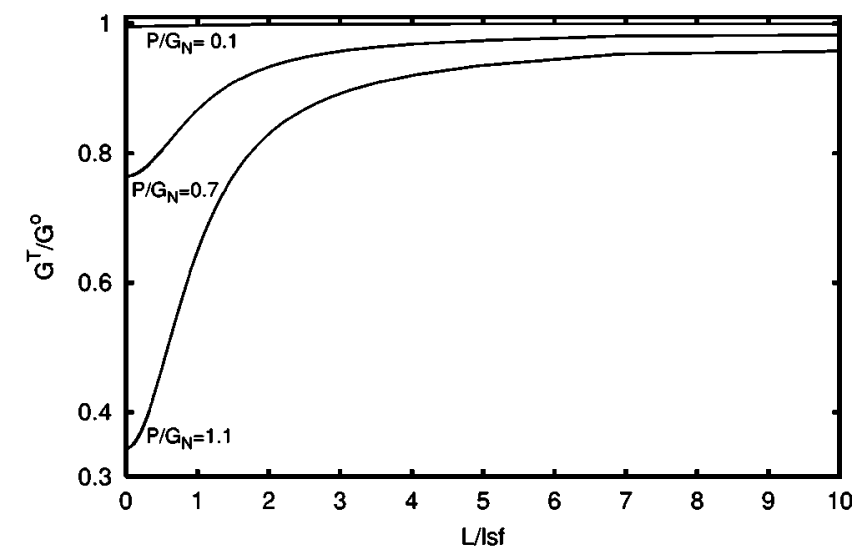

(b)

FIG. 5. Effect of spin-flip scattering on the system: For symmetric contacts and zero magnetic field. (a) $G^{T}$ normalized to $G^{0}$ as a function of $L / l_{s f}$, for the following set of parameters: $G^{\uparrow} / G_{N}$ $=1.0, G^{\downarrow} / G_{N}=0.3, \operatorname{Re} G^{\uparrow \downarrow} / G_{N}=0.7, \operatorname{Im} G^{\uparrow \downarrow} / G_{N}=0.0$. (b) $G^{T} / G^{0}$ versus $L / l_{s f}$ in the case of antiparallel configuration, for different values of the relative polarization $P / G_{N}=0.1,0.7,1.1$, and for $G / G_{N}=1.3$ constant.

case the conductance approaches $G^{0}$ asymptotically in different ways, depending on the magnitude of the spin accumulation.

\section{Effect of the magnetic field: Precession and relaxation}

In a diffusive system the presence of an external magnetic field relaxes the spin accumulation, in addition to the usual precession of the spin. Semiclassically, the spin accumulation at a certain position $x$ is the average contribution of the spin of all electrons. In a diffusive metal each electron diffuses along a random trajectory, while its spin precesses with frequency $\omega_{L}$ around the magnetic field. Since each trajectory has a different length, the spins of the electrons at a certain point $x$ are oriented in different directions, which in average relaxes the local spin polarization. The length scale of both relaxation and precession processes is the precession length $l_{B}=\sqrt{2 \hbar D / g \mu_{B} B}$, where $D$ is the diffusion coefficient, $B$ is the magnetic field, $\mu_{B}$ is the Bohr magneton and $g$ is the spin gyromagnetic ratio.

The external magnetic field may also influence the transport processes described by the mixing conductance $G^{\uparrow \downarrow}$ at the contacts. Let us consider for simplicity that $G_{N}$
$\gg\left(G^{\uparrow}, G^{\downarrow}, G^{\uparrow \downarrow}\right)$, and $l_{s f} \rightarrow \infty$. In this limit the distribution function of the normal metal does not depend on position. From current conservation we have

$$
\begin{gathered}
i_{\mathcal{L}}^{C}+i_{\mathcal{R}}^{C}=0, \\
\vec{i}_{\mathcal{L}}^{C}+\vec{i}_{\mathcal{R}}^{C}=\left(\frac{g \mu_{B}}{\hbar} \vec{B} \times \vec{f}\right) V_{o l},
\end{gathered}
$$

where Eq. (25) corresponds to the particle current, Eq. (26) corresponds to the spin current and $V_{o l}$ is the volume of the normal metal. The current is defined to be positive when injected into the normal metal by the ferromagnetic reservoirs. We can rewrite Eq. (26) as

$$
\vec{i}_{\mathcal{L}}^{C}+\vec{i}_{\mathcal{R}}^{C}=\left(g \vec{\omega}_{L} \times \vec{f}\right) V_{o l},
$$

where $\vec{\omega}_{L}$ is the Larmor frequency vector. From this expression follows that the time scale relevant for $\omega_{L}$ is the escape time $\tau_{e s c}=e^{2} \nu_{D O S} . V_{o l} / G^{\text {contact }}$, where $G^{\text {contact }}$ is the average contact conductance. $\tau_{\text {esc }}$ is the time in which an electron escapes from the normal metal into the ferromagnetic reservoirs. It is also the time scale relevant for the precession of the electrons around the magnetic field. On the other hand, if $G_{N} \sim\left(G^{\uparrow}, G^{\downarrow}, G^{\uparrow \downarrow}\right), \tau_{e s c}$ is of the order of the Thouless time $\tau_{D}$, which is the average time in which an electron passes through the diffusive normal metal. When $G_{N}$ $\sim\left(G^{\uparrow}, G^{\downarrow}, G^{\uparrow \downarrow}\right)$ diffusion, precession and transmission or reflection at the contacts, happen on the same time scale. From these estimates we see that the ballistic or diffusive nature of the normal metal is not going to change the effect of the magnetic field on the physics at the contacts. The results obtained for $G_{N} \gg\left(G^{\uparrow}, G^{\downarrow}, G^{\uparrow \downarrow}\right)$, should therefore be valid when $G_{N} \sim\left(G^{\uparrow}, G^{\downarrow}, G^{\uparrow \downarrow}\right)$. We now make a perturbation expansion in small magnetic fields (see the Appendix). To first order, the current depends on the expansion parameter $B$ as

$$
\frac{\partial i_{0}}{\partial B}=\vec{s} \hat{\mathbf{C}}^{-1} \hat{\mathbf{M}} \hat{\mathbf{C}}^{-1} \vec{b}
$$

where $\vec{s}$ and $\vec{b}$ are vectors associated with the spin current injected into the normal metal (see the Appendix) and where the matrix $\hat{\mathbf{C}}$ describes the contacts and $\hat{\mathbf{M}}$ the magnetic-field contribution. As detailed in the Appendix, $\hat{\mathbf{C}}$ has a symmetric part $\hat{\mathbf{S}}_{\hat{\mathbf{C}}}$, which only includes three of the four contact conductances, i.e., only the conductances $G^{\uparrow}, G^{\downarrow}, \operatorname{Re} G^{\uparrow \downarrow}$ of each contact ( $\mathcal{L}$ and $\mathcal{R}$ ), respectively. On the other hand, $\hat{\mathbf{C}}$ has also an antisymmetric part which only depends on the imaginary part of the mixing conductance of each contact $\operatorname{Im} G_{\mathcal{L}, \mathcal{R}}^{\uparrow \downarrow}$. The matrix $\hat{\mathbf{M}}$, which describes the precession of spins due to the magnetic field, is also antisymmetric. Using the symmetry properties of the matrices $\hat{\mathbf{C}}$ and $\hat{\mathbf{M}}$ we can determine from Eq. (27) the symmetry properties of the total conductance of the system $G^{T}=i_{0} /\left(f_{\mathcal{L}}^{F}-f_{\mathcal{R}}^{F}\right)$ with respect to the magnetic field $B$.

When $\hat{\mathbf{C}}$ is a symmetric matrix, $\partial G^{T} / \partial B=0$ for small values of magnetic field. The conductance of the system is then symmetric with respect to a change of sign of the magnetic field $(\vec{B} \rightarrow-\vec{B})$, i.e., with respect to time reversal. On the other hand, if $\hat{\mathbf{C}}$ is antisymmetric, $\partial G^{T} / \partial B \neq 0$ and we 


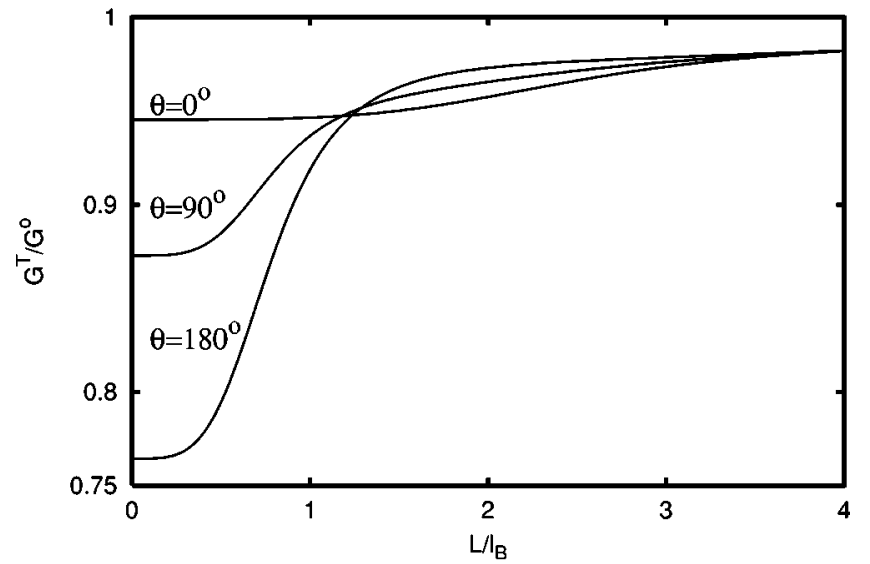

(a)

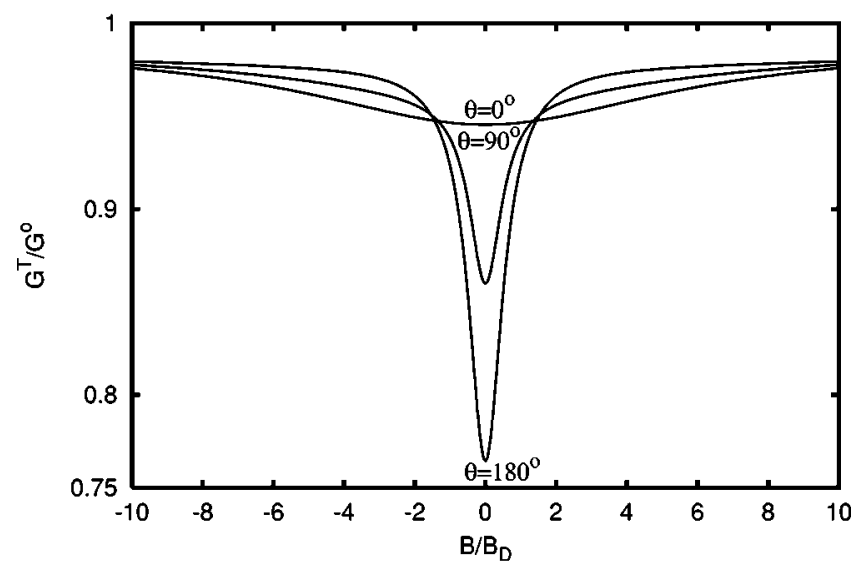

(b)

FIG. 6. Magnetic-field dependence in the absence of spin-flip scattering: We consider symmetric contacts and the following set of parameters: $G^{\uparrow} / G_{N}=1.0, G^{\downarrow} / G_{N}=0.3, \operatorname{Re} G^{\uparrow \downarrow} / G_{N}=0.7$, $\operatorname{Im} G^{\uparrow \downarrow} / G_{N}=0.5$. Moreover, the magnetic field is always perpendicular to both magnetizations directions $\vec{B} \cdot \vec{m}_{\mathcal{L}, \mathcal{R}}=0$. (a) $G^{T} / G^{0}$ as a function of $L / l_{B}$; (b) $G^{T} / G^{0}$ versus $B / B_{D}$.

can expect asymmetric behavior of the conductance with respect to change of sign of magnetic field.

\section{Modulation of the conductance by the magnetic field: Symmetry with respect to time reversal}

In the following, we discuss the dependence of the conductance on the magnetic field. We obtain $G^{T} / G^{0}$ as a function of $L / l_{B} \sim L \sqrt{B}$ for different magnetic configurations, $l_{s f} \rightarrow \infty$ and $L$ constant. In Fig. 6(a) we plot $G^{T} / G^{0}$ in the case of symmetric contacts, where the magnetic field is perpendicular to both magnetization directions $\vec{B} \cdot \vec{m}_{\mathcal{L}, \mathcal{R}}=0$. In this case all injected spins precess around the magnetic field. When $l_{B} \gg L$, the spins injected from one ferromagnet are not strongly affected by the magnetic field, so they travel through the normal metal and reach the other ferromagnet without relaxation. As a result, the total conductance depends on the relative magnetic configuration. By decreasing $l_{B}$, the spin accumulation precesses and relaxes on the scale of $l_{B}$. Due to the precession of spins, the conductance displays in general a nonmonotonic behavior with $L / l_{B}$. This modulation of the conductance can be understood in terms of the "matching" of the spins at the contacts after precession.
According to the values of the contact conductances for the different magnetic configurations the spins are reflected or transmitted at the contacts depending on its orientation. Concerning the relaxation, the configuration with more spin accumulation (in this case, the antiparallel configuration) is the most sensitive to the magnetic field (increases faster than the other ones), since there are more spins to be rotated by the magnetic field in this configuration than in others. In Fig. 6(a), the conductance of $\theta=180^{\circ}$ (antiparallel) and $\theta=90^{\circ}$ configurations cross the conductance for $\theta=0^{\circ}$ (parallel) around $L / l_{B}=1$. That means that at this point the spins accumulated in these two configurations have been reduced to the value of spin accumulation of $\theta=0^{\circ}$ configuration. After the point $L / l_{B}=1$, the parallel configuration $\left(\theta=0^{\circ}\right)$ gives a smaller conductance and the antiparallel configuration $(\theta$ $=180^{\circ}$ ) gives the highest conductance. As a result, for $L / l_{B}>1$, the parallel configuration is more sensitive to the magnetic field than the antiparallel configuration (now the one which increases faster). The relaxation of spins via the precession around the magnetic field depends on the amount of spin accumulation in the system. This nonmononotic behavior of the conductance is specially relevant between parallel and antiparallel configurations, because the difference between the conductance of both configurations $G_{P}^{T}-G_{A P}^{T}$ can be modulated from positive to negative values by the external magnetic field.

When $\vec{B} \cdot \vec{m}_{\mathcal{L}, \mathcal{R}}=0$, according to Eq. (A7) in the Appen$\operatorname{dix}, \vec{B}$ has only one component $B_{3} \vec{\omega} \sim B_{3}\left(\vec{m}_{\mathcal{L}} \times \vec{m}_{\mathcal{R}}\right)$ (see Ref. 22). Moreover, the spins are injected with directions along $\vec{m}_{\mathcal{L}}$ and $\vec{m}_{\mathcal{R}}$, so the precession due to the magnetic field only switches the spin directions between $\vec{m}_{\mathcal{L}}$ and $\vec{m}_{\mathcal{R}}$. As a result, the distribution function given by Eq. (A3), has only two components $\vec{f}=f_{1} \vec{u}+f_{2} \vec{v}$. In this particular case, $\hat{\mathbf{C}}$ reduces to $\hat{\mathbf{S}}_{\hat{\mathbf{C}}}$, which is a symmetric matrix. The same holds for the matrix $\hat{\mathbf{M}}$, which reduces to its $3 \times 3$ upper box, which only includes $B_{3}$ (see the Appendix). As $\hat{\mathbf{C}}$ reduces to $\hat{\mathbf{S}}_{\hat{\mathbf{C}}}$, we expect $\partial G^{T} / \partial B=0$. Figure $6(\mathrm{~b})$ shows the dependence of $G^{T} / G^{0}$ on $B / B_{D}$ for different magnetic configurations, where $B_{D}=2 \hbar / g \mu_{B} \tau_{D}$ is the scale of magnetic fields relevant for precession in a diffusive medium. As expected, all configurations are symmetric with respect to a change of sign in magnetic field $(\vec{B} \rightarrow-\vec{B})$.

\section{Modulation of the conductance by the magnetic field: Asymmetric properties with respect to time reversal}

Now we want to investigate the role of $\operatorname{Im} G^{\uparrow \downarrow}$. To this end, the magnetic field is assumed to be oriented perpendicular to both magnetizations when the system is in collinear configurations, and parallel to the direction of one of the magnetizations when the system is in the $\theta=90^{\circ}$ configuration. According to Eq. (A7) in the Appendix, for $\theta=90^{\circ}$, the magnetic field is along $B_{1} \vec{u}+B_{2} \vec{v}$, and as a result from the injection and precession, there are spins in the three directions $\vec{f}=f_{1} \vec{u}+f_{2} \vec{v}+f_{3} \vec{\omega}$, i.e., the precession of spins around the magnetic field induces spins along the perpendicular direction $\left(\vec{m}_{\mathcal{L}} \times \vec{m}_{\mathcal{R}}\right)$ to the injection orientations $\vec{m}_{\mathcal{L}}$ and $\vec{m}_{\mathcal{R}}$. $\hat{\mathbf{C}}$ is then an antisymmetric matrix, due to the contributions of the terms which includes $\operatorname{Im} G^{\uparrow \downarrow}$. So for $\operatorname{Im} G^{\uparrow \downarrow} \neq 0$, 


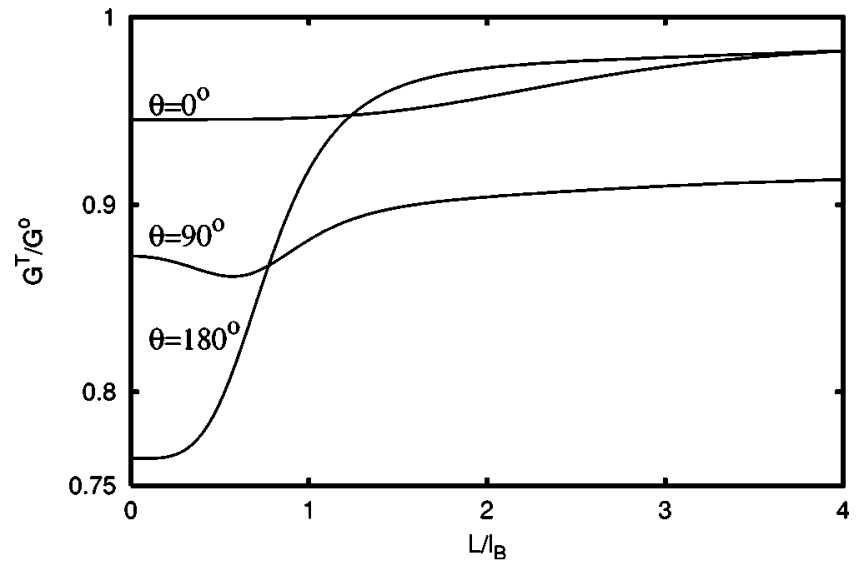

(a)

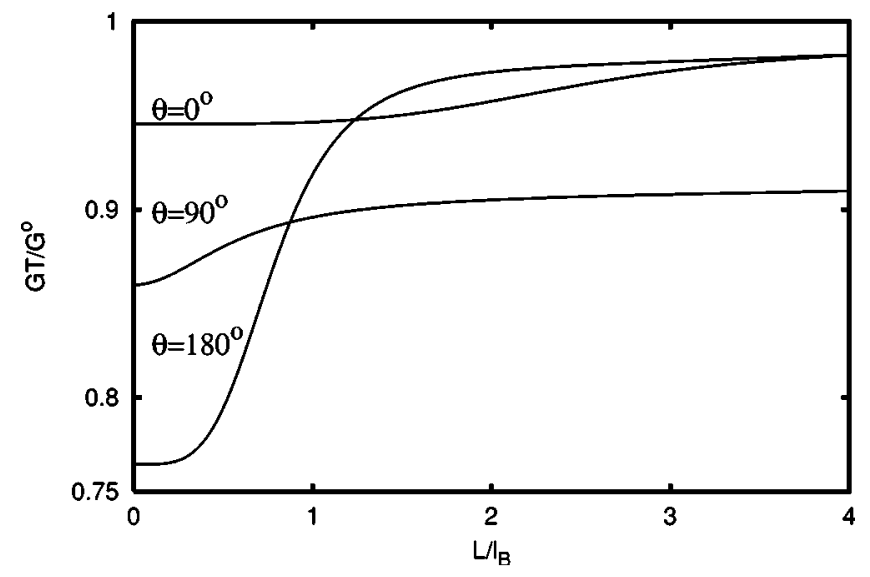

(b)

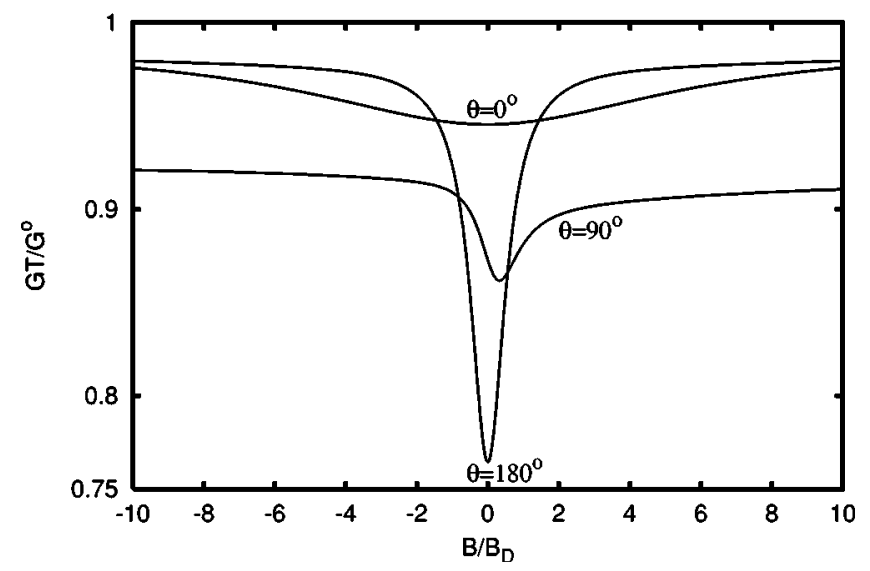

(c)

FIG. 7. Magnetic-field dependence in the absence of spin-flip scattering: We consider $\vec{B} \cdot \vec{m}_{\mathcal{L}}=0, \vec{B} \cdot \vec{m}_{\mathcal{R}}^{0^{\circ}, 180^{\circ}}=0$, and $\vec{B} \| \vec{m}_{\mathcal{R}}^{90^{\circ}}$ (or $\vec{B} \cdot \vec{m}_{\mathcal{R}}=0, \vec{B} \cdot \vec{m}_{\mathcal{L}}^{0^{\circ}, 180^{\circ}}=0$, and $\left.\vec{B} \| \vec{m}_{\mathcal{L}}^{90^{\circ}}\right)$. (a) $G^{T} / G^{0}$ vs $L / l_{B}$, for symmetric contacts and the following set of parameters: $G^{\uparrow} / G_{N}$ $=1.0, G^{\downarrow} / G_{N}=0.3, \operatorname{Re} G^{\uparrow \downarrow} / G_{N}=0.7, \operatorname{Im} G^{\uparrow \downarrow} / G_{N}=0.5$. (b) Same as (a) but for $\operatorname{Im} G^{\uparrow \downarrow} / G_{N}=0.0$. (c) $G^{T} / G^{0}$ versus $B / B_{D}$, for $G^{\uparrow} / G_{N}=1.0, G^{\downarrow} / G_{N}=0.3, \operatorname{Re} G^{\uparrow \downarrow} / G_{N}=0.7, \operatorname{Im} G^{\uparrow \downarrow} / G_{N}=0.5$.

$\partial G^{T} / \partial B \neq 0$, which means asymmetric behavior of the conductance with respect to time reversal. On the other hand, if we put $\operatorname{Im} G^{\uparrow \downarrow}=0, \hat{\mathbf{C}}$ is symmetric and $\partial G^{T} / \partial B=0$.

In Fig. 7(a) we obtain $G^{T} / G^{0} v s L / l_{B}$, for the same set of parameters as in Fig. 6(a). For parallel and antiparallel configurations, the results are not modified compared to Fig.

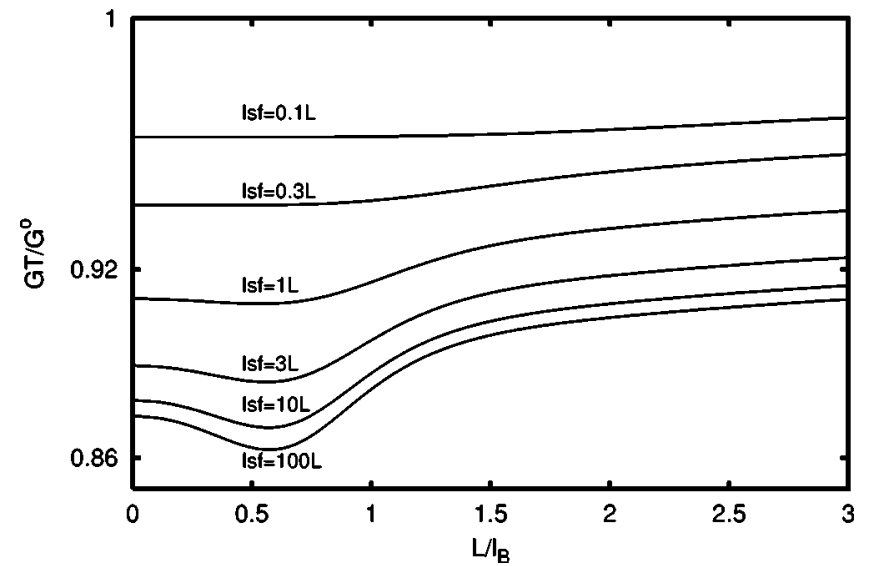

FIG. 8. Magnetic-field dependence and spin-flip scattering: Conductance for $\theta=90^{\circ}$ configuration of Fig. 7(a), for different ratios $l_{s f} / L=0.1,0.3,1,3,10,100$.

6(a). However, for $\theta=90^{\circ}$ the relative conductance $G^{T} / G^{0}$ does not approach unity asymptotically. In this configuration there are some injected spins, which are parallel to the magnetic field and which do not precess at all. So this part of the spin accumulation remains in the system and does not relax irrespective of the values of the magnetic field. More interesting is the appearance of a dip in the conductance for small values of the magnetic field. If we repeat the calculation of $G^{T} / G^{0}$ vs $L / l_{B}$ for the same set of parameters except for $\operatorname{Im} G^{\uparrow \downarrow} / G_{N}=0$, we see that the dip disappears [Fig. 7(b)], so according to our discussion, it is related with asymmetric properties of the conductance. In Fig. 7(c), we plot $G^{T} / G^{0}$ vs $B / B_{D}$. As we expect, $\theta=90^{\circ}$ configuration presents asymmetric behavior respect time reversal, whereas both parallel and antiparallel configurations remain symmetric [Fig. 7(c)]. In particular the $\theta=90^{\circ}$ conductance is antisymmetric with respect to time reversal, for small values of magnetic field.

From this discussion, we understand that the real part of the mixing conductance describes processes at the contacts in which spins perpendicular to the magnetization direction, are transmitted or reflected obeying timer-eversal symmetry. On the other hand, the imaginary part of the mixing conductance describes processes in which the spins precess around the magnetization vector of the ferromagnet. As a result of the precession, the orientation of the spin changes. The latter processes are antisymmetric with respect to time reversal.

\section{Supression of the magnetic-field effects by spin-flip scattering}

Spin-flip scattering causes relaxation of the spin accumulation in the normal metal and as a result, suppression of the spin-dependent properties on the system. Now we want to investigate how the spin-flip affect the magnetic-field effects shown above. The existence of spin-flip scattering reduces $l_{s f}$. If $l_{s f} \gg L$, there is no strong spin-flip scattering in the system and it is possible to observe spin-dependent effects. On the other hand, if $l_{s f} \ll L$, the injected spins relax very fast due to spin-flip processes and no spin-dependent effects can be observed. In particular in Fig. 8 we show how the dip of $\theta=90^{\circ}$ configuration from Figs. 7(a) and 7(c), is suppressed by spin-flip scattering on the system. Also by decreasing $l_{s f}, G^{T} / G^{0}$ increases for constant magnetic field, to the value 
1. That simply means, that the spin accumulation relaxes due to spin-flip scattering, as is expected.

\section{DISCUSSION AND CONCLUSIONS}

In this paper, the normal metal in our FNF device is considered three dimensional, but can also be two dimensional (2D), e.g., a two-dimensional electron gas (2DEG) attached to ferromagnetic reservoirs, or even one dimensional (1D), if the normal metal is a quantum wire or a carbon nanotube. ${ }^{23}$ In this case electron-electron interaction should be taken into account. $^{24}$ The nonmagnetic material can also be a semiconductor, as shown in recent spin-injection experiments. ${ }^{25}$ In the case of a $2 \mathrm{DEG}$ attached to metallic ferromagnets, the large difference between the conductivities of the 2DEG and the ferromagnetic reservoirs suppresses the spin injection via metallic contacts. For a significant spin injection into the 2DEG, tunnel contacts, a semiconductor ferromagnet or a half metallic ferromagnet are required. ${ }^{26}$

In this paper we have shown how the spin-dependent transport through a FNF double heterojunction can be described in terms of the spin-dependent conductances of the contacts $\left(G^{\uparrow}, G^{\downarrow}, G^{\uparrow \downarrow}\right)$, the magnetization direction $\vec{m}$ of the ferromagnetic reservoirs, and the normal-metal conductance $G_{N}$. The dependence of the conductance on the relative angle between the magnetizations of the different ferromagnets is affected by the mixing conductance $G^{\uparrow \downarrow}$. For noncollinear transport between the ferromagnetic reservoirs, $G^{\uparrow \downarrow}$ $=\operatorname{Re} G^{\uparrow \downarrow}+i \operatorname{Im} G^{\uparrow \downarrow}$ describes transport of spins perpendicular to the magnetization direction of the ferromagnets. These processes enhance the conductance for noncollinear configurations, which may be used in multiterminal devices for modulation of the transport properties. ${ }^{9}$ This modulation could be useful for future applications as spin-dependent transistors. We find that spin injection can be symmetric and antisymmetric with respect to time reversal. The symmetric processes are described by $\operatorname{Re} G^{\uparrow \downarrow}$ and the antisymmetric ones are described by $\operatorname{Im} G^{\uparrow \downarrow}$. It is interesting to observe that the antisymmetric processes described by $\operatorname{Im} G^{\uparrow \downarrow}$ correspond to spin precession around the magnetization vector of the ferromagnet which couples to an external magnetic field.

In a diffusive system, an applied magnetic field produces both precession and relaxation of the spin accumulation. The conductance displays a nonmonotonic behavior on the scale of the precession length $l_{B}$, which is the distance for the precession of the spin around the magnetic field in the normal metal. Due to this modulation, the difference between the conductances of the parallel and antiparallel configurations $G_{P}^{T}-G_{A P}^{T}$ can be positive and negative as a function of the magnetic field. A possible candidate to observe this effect is $\mathrm{Al}$, which has a large $l_{s f}$ and which can be coupled to ferromagnetic reservoirs (e.g., $\mathrm{Fe}, \mathrm{CoFe}, \mathrm{NiFe}, \mathrm{Co}, . .$. ) via metallic junctions or also $\mathrm{Al}_{2} \mathrm{O}_{3}$ tunnel junctions. Let us estimate the values of magnetic fields for $\mathrm{Al}$ single crystal associated with the points $L / l_{B}=0.5$ and $L / l_{B}=2$ of Fig. $6(\mathrm{a})$, where $G_{P}^{T}-G_{A P}^{T}$ is positive and negative, respectively. If the length of the system is $L=10 \mu \mathrm{m}$, which is comparable with the spin-diffusion length $\left(L \sim l_{s f}=10-70 \mu \mathrm{m}\right){ }^{5}$ we obtain for $L / l_{B}=0.5: B^{(+)} \sim 0.01 \mathrm{~T}$ and for $L / l_{B}$ $=2: B^{(-)} \sim 0.1$ T. If $L=1 \mu \mathrm{m}\left(L \ll l_{s f}\right)$, we obtain for
$L / l_{B}=0.5: B^{(+)} \sim 0.1 \mathrm{~T}$ and for $L / l_{B}=2: B^{(-)} \sim 1 \mathrm{~T}$. In both cases we see that the values of magnetic field in the case of Al single crystal are quite reasonable and also that the change from positive to negative $G_{P}^{T}-G_{A P}^{T}$ can be achieved by an increase of the magnetic field by one order of magnitude. The same estimate for polycrystalline Al gives us for $L \sim l_{s f}=0.7 \mu \mathrm{m},{ }^{19}$ the values of $B^{(+)} \sim 1 \mathrm{~T}$ and $B^{(-)}$ $\sim 16 \mathrm{~T}$, respectively. These fields are much higher than the typical switching field for a ferromagnet, so polycrystalline Al does not appear to be a good candidate. For very pure $\mathrm{Na}$, if $L \sim l_{s f}=0.4 \mathrm{~cm}$, the corresponding values of magnetic fields are $B^{(+)} \sim 1 \mu \mathrm{T}$ and $B^{(-)} \sim 10 \mu \mathrm{T}$, respectively. This modulation of $G_{P}^{T}-G_{A P}^{T}$ by a magnetic field can also be explored in semiconductors (SC) 2DEG, as, e.g., GaAs and InAs. When $l_{B} \sim l_{s f}$ the following expression holds for the magnetic field corresponding to $L / l_{B}=1: B=2(\hbar /$ $\left.\mu_{B}\right)\left(\tau_{s f} g\right)^{-1}=2.27 \times 10^{-11}\left(\tau_{s f} g\right)^{-1}$, which depends on the spin-flip time $\tau_{s f}$ and on the gyromagnetic ratio $g$ of the semiconductor material. For SC, $g$ depends strongly on the material (e.g., $g^{G a A s}=-0.4, g^{\operatorname{InAs}}=15.0$ ), so depending on the values of $\tau_{s f}$, one can obtain the corresponding values of magnetic field. Kikkawa and Awschalom ${ }^{27}$ report $\tau_{s f}$ $\sim 10^{-7} s$ in $n$-type GaAs system, but this value corresponds to spin lifetimes of optically pumped carriers, and not to the usual carriers relevant for transport. The corresponding value for the magnetic field for this case is $B^{G a A s} \sim 510^{-4} \mathrm{~T}$. On the other hand, we are not aware of reliable values of $\tau_{s f}$ for transport in these systems. In conclusion, from our estimates of the relevant values of magnetic fields, Al single crystals with ferromagnetic contacts are good candidates to test our predictions and possibly lead to the discovery of other new physical phenomena of spin transport.

\section{ACKNOWLEDGMENTS}

This work is part of the research program for the "Stichting voor Fundamenteel Onderzoek der Materie', (FOM). We acknowledge support from the NEDO Joint Research Program (NTDP-98). It is a pleasure to acknowledge useful discussions with W. Belzig, D. Pfannkuche, and Y. Tokura.

\section{APPENDIX: PERTURBATION EXPANSION IN SMALL MAGNETIC FIELDS}

Equations (25) and (26) can be written as follows:

$$
\left(\frac{G_{\mathcal{L}}}{2}+\frac{G_{\mathcal{R}}}{2}\right) f_{0}+\frac{P_{\mathcal{L}}}{2}\left(\vec{f} \vec{m}_{\mathcal{L}}\right)+\frac{P_{\mathcal{R}}}{2}\left(\vec{f} \cdot \vec{m}_{\mathcal{R}}\right)=\frac{G_{\mathcal{L}}}{2} f_{\mathcal{L}}^{F}+\frac{G_{\mathcal{R}}}{2} f_{\mathcal{R}}^{F}
$$

$$
\begin{aligned}
\left(\frac{G_{\mathcal{L}}}{2}-\right. & \left.\operatorname{Re} G_{\mathcal{L}}^{\uparrow \downarrow}\right)\left(\vec{f} \cdot \vec{m}_{\mathcal{L}}\right) \vec{m}_{\mathcal{L}}+\operatorname{Re} G_{\mathcal{L}}^{\uparrow \downarrow} \vec{f}+\operatorname{Im} G_{\mathcal{L}}^{\uparrow \downarrow}\left(\vec{f} \times \vec{m}_{\mathcal{L}}\right) \\
& +\left(\frac{G_{\mathcal{R}}}{2}-\operatorname{Re} G_{\mathcal{R}}^{\uparrow \downarrow}\right)\left(\vec{f} \cdot \vec{m}_{\mathcal{R}}\right) \vec{m}_{\mathcal{R}}+\operatorname{Re} G_{\mathcal{R}}^{\uparrow \downarrow} \vec{f} \\
& +\operatorname{Im} G_{\mathcal{R}}^{\uparrow \downarrow}\left(\vec{f} \times \vec{m}_{\mathcal{R}}\right) \\
= & \frac{P_{\mathcal{L}}}{2}\left(f_{\mathcal{L}}^{F}-f_{0}\right) \vec{m}_{\mathcal{L}}+\frac{P_{\mathcal{R}}}{2}\left(f_{\mathcal{R}}^{F}-f_{0}\right) \vec{m}_{\mathcal{R}}-\left(\frac{g \mu_{B}}{\hbar} \vec{B} \times \vec{f}\right) V_{o l} .
\end{aligned}
$$


Now we expand $\vec{f}$ into a convenient basis of the vectors $\vec{m}_{\mathcal{L}}, \vec{m}_{\mathcal{R}}$, and $\vec{m}_{\mathcal{L}} \times \vec{m}_{\mathcal{R}}$ as

$$
\vec{f}=f_{1} \vec{u}+f_{2} \vec{v}+f_{3} \vec{\omega},
$$

where

$$
\begin{aligned}
& \vec{u}=\frac{\vec{m}_{\mathcal{L}}+\vec{m}_{\mathcal{R}}}{\sqrt{2(1+m)}}, \\
& \vec{v}=\frac{\vec{m}_{\mathcal{L}}-\vec{m}_{\mathcal{R}}}{\sqrt{2(1+m)}},
\end{aligned}
$$

In terms of this expansion, we can combine Eqs. (A1) and (A2) into a compact matrix form as

and where $m=\vec{m}_{\mathcal{L}} \cdot \vec{m}_{\mathcal{R}}=\cos \theta$. We can also express the magnetic field in this basis as

$$
\vec{B}=B_{1} \vec{u}+B_{2} \vec{v}+B_{3} \vec{\omega} .
$$

$$
(\hat{\mathbf{C}}+\hat{\mathbf{M}}) \vec{a}=\vec{b},
$$

where

$$
\begin{aligned}
& \hat{\mathbf{C}}=\left(\begin{array}{cccc}
\cdot & \cdot & \cdot & 0 \\
\cdot \hat{\mathbf{S}}_{\hat{\mathbf{C}}} & \cdot & \sqrt{\frac{1-m}{2}}\left(\operatorname{Im} G_{\mathcal{L}}^{\uparrow \downarrow}-\operatorname{Im} G_{\mathcal{R}}^{\uparrow \downarrow}\right) \\
\cdot(3 \times 3) & \cdot & -\sqrt{\frac{1+m}{2}}\left(\operatorname{Im} G_{\mathcal{L}}^{\uparrow \downarrow}+\operatorname{Im} G_{\mathcal{R}}^{\uparrow \downarrow}\right) \\
0 & -\sqrt{\frac{1-m}{2}}\left(\operatorname{Im} G_{\mathcal{L}}^{\uparrow \downarrow}-\operatorname{Im} G_{\mathcal{R}}^{\uparrow \downarrow}\right) & \sqrt{\frac{1+m}{2}}\left(\operatorname{Im} G_{\mathcal{L}}^{\uparrow \downarrow}+\operatorname{Im} G_{\mathcal{R}}^{\uparrow \downarrow}\right) & \operatorname{Re} G_{\mathcal{L}}^{\uparrow \downarrow}+\operatorname{Re} G_{\mathcal{R}}^{\uparrow \downarrow}
\end{array}\right), \\
& \hat{\mathbf{S}}_{\hat{\mathbf{C}}}=\left(\begin{array}{ccc}
\frac{G_{\mathcal{L}}+G_{\mathcal{R}}}{2} & \sqrt{\frac{1+m}{2} \frac{P_{\mathcal{L}}+P_{\mathcal{R}}}{2}} & \sqrt{\frac{1-m}{2}} \frac{P_{\mathcal{L}}-P_{\mathcal{R}}}{2} \\
\sqrt{\frac{1+m}{2}} \frac{P_{\mathcal{L}}+P_{\mathcal{R}}}{2} & \frac{\left(G_{\mathcal{L}}+G_{\mathcal{R}}\right)(1+m)}{4}+\frac{\left(\operatorname{Re} G_{\mathcal{L}}^{\uparrow \downarrow}+\operatorname{Re} G_{\mathcal{R}}^{\uparrow \downarrow}\right)(1-m)}{2} & \left(\frac{G_{\mathcal{L}}-G_{\mathcal{R}}}{2}-\operatorname{Re} G_{\mathcal{L}}^{\uparrow \downarrow}+\operatorname{Re} G_{\mathcal{R}}^{\uparrow \downarrow}\right)\left(\frac{\sqrt{1-m^{2}}}{2}\right) \\
\sqrt{\frac{1-m}{2}} \frac{P_{\mathcal{L}}-P_{\mathcal{R}}}{2} & \left(\frac{G_{\mathcal{L}}-G_{\mathcal{R}}}{2}-\operatorname{Re} G_{\mathcal{L}}^{\uparrow \downarrow}+\operatorname{Re} G_{\mathcal{R}}^{\uparrow \downarrow}\right)\left(\frac{\sqrt{1-m^{2}}}{2}\right) & \frac{\left(G_{\mathcal{L}}+G_{\mathcal{R}}\right)(1-m)}{4}+\frac{\left(\operatorname{Re} G_{\mathcal{L}}^{\uparrow \downarrow}+\operatorname{Re} G_{\mathcal{R}}^{\uparrow \downarrow}\right)(1+m)}{2}
\end{array}\right),
\end{aligned}
$$

$(\mathrm{A} 8 \mathrm{c})$

and where

$$
\begin{gathered}
\hat{\mathbf{M}}=V_{o l} \frac{g \mu_{B}}{\hbar}\left(\begin{array}{cccc}
0 & 0 & 0 & 0 \\
0 & 0 & B_{3} & -B_{2} \\
0 & -B_{3} & 0 & B_{1} \\
0 & B_{2} & -B_{1} & 0
\end{array}\right), \\
\vec{a}=\left(\begin{array}{l}
f_{0} \\
f_{1} \\
f_{2} \\
f_{3}
\end{array}\right)
\end{gathered}
$$




$$
\vec{b}=\frac{1}{2}\left(\begin{array}{c}
G_{\mathcal{L}} f_{\mathcal{L}}^{F}+G_{\mathcal{R}} f_{\mathcal{R}}^{F} \\
\sqrt{\frac{1+m}{2}}\left(P_{\mathcal{L}} f_{\mathcal{L}}^{F}+P_{\mathcal{R}} f_{\mathcal{R}}^{F}\right) \\
\sqrt{\frac{1-m}{2}}\left(P_{\mathcal{L}} f_{\mathcal{L}}^{F}-P_{\mathcal{R}} f_{\mathcal{R}}^{F}\right) \\
0
\end{array}\right) .
$$

By a perturbation expansion in small magnetic fields, we may study how the magnetic field is coupled with the physics at the contacts. To zeroth order in magnetic field we simply have

$$
\vec{a}^{(0)}=\hat{\mathbf{C}}^{-1} \vec{b}
$$

To first order

$$
\vec{a}^{(1)}=\left(\hat{\mathbf{C}}^{-1} \hat{\mathbf{M}} \hat{\mathbf{C}}^{-1}\right) \vec{b}
$$

The total particle current in the system is given by

$$
i_{0}=i_{\mathcal{L}}^{C}-i_{\mathcal{R}}^{C}=\vec{s} \cdot \vec{a}+G_{\mathcal{L}} f_{\mathcal{L}}^{F}+G_{\mathcal{R}} f_{\mathcal{R}}^{F},
$$

where

$$
\vec{s}=\left(\begin{array}{c}
-G_{\mathcal{L}}+G_{\mathcal{R}} \\
\sqrt{\frac{1+m}{2}}\left(-P_{\mathcal{L}}+P_{\mathcal{R}}\right) \\
-\sqrt{\frac{1-m}{2}}\left(P_{\mathcal{L}}+P_{\mathcal{R}}\right) \\
0
\end{array}\right) .
$$

The dependence of the current on the expansion parameter $B$ is given by

$$
\frac{\partial i_{0}}{\partial B}=\vec{s} \cdot \vec{a}
$$

which to first order reduces to

$$
\frac{\partial i_{0}}{\partial B}=\vec{s} \cdot \vec{a}(1)=\vec{s} \hat{\mathbf{C}}^{-1} \hat{\mathbf{M}} \hat{\mathbf{C}}^{-1} \vec{b} .
$$

${ }^{1}$ M.N. Baibich, J.M. Broto, A. Fert, F. Nguyen Van Dau, F. Petroff, P. Etienne, G. Creuzet, A. Friederich, and J. Chazelas, Phys. Rev. Lett. 61, 2472 (1988); G. Binach, P. Grunberg, F. Saurenbach, and W. Zinn, Phys. Rev. B 39, 4828 (1989).

${ }^{2}$ R.E. Camley and J. Barnas, Phys. Rev. Lett. 63, 664 (1989); M. Levy, S. Zhang, and A. Fert, ibid. 65, 1643 (1990); S.F. Lee, W.P. Pratt, Q. Yang, P. Holody, R. Loloee, P.A. Schroeder, and J. Bass, J. Magn. Magn. Mater. 118, 1 (1993); P.M. Levy, Solid State Phys. 47, 367 (1994); M.A.M. Gijs and G.E.W. Bauer, Adv. Phys. 46, 285 (1997).

${ }^{3}$ J.C. Slonczewski, Phys. Rev. B 39, 6995 (1989); V.V. Ustinov and E.A. Kravtsov, J. Phys.: Condens. Matter 7, 3471 (1995); H.E. Camblong, P.M. Levy, and S. Zang, Phys. Rev. B 51, 16052 (1995); A. Vedyayev, N. Ryzhanova, B. Dieny, P. Dauguet, P. Gandit, and J. Chaussy, ibid. 55, 3728 (1997).

${ }^{4}$ P. Dauguet, P. Gandit, J. Chaussy, S.F. Lee, A. Fert, and P. Holody, Phys. Rev. B 54, 1083 (1996).

${ }^{5}$ M. Johnson and R.H. Silsbee, Phys. Rev. Lett. 55, 1790 (1985); Phys. Rev. B 37, 5326 (1988); M. Johnson, Phys. Rev. Lett. 70, 2142 (1993).

${ }^{6}$ M. Johnson, Science 260, 320 (1993).

${ }^{7}$ K. Ono, H. Shimada, S. Kobayashi, and Y. Ootuka, J. Phys. Soc. Jpn. 65, 3449 (1996).

${ }^{8}$ J. Barnas and A. Fert, Phys. Rev. Lett. 80, 1058 (1998); S. Takahashi and S. Maekawa, ibid. 80, 1758 (1998); A. Brataas, Yu.V. Nazarov, J. Inoue, and G.E.W. Bauer, Phys. Rev. B 59, 93 (1999); X.H. Wang and A. Brataas, Phys. Rev. Lett. 83, 5138 (1999); J. Barnas and A. Fert, J. Magn. Magn. Mater. 192, 391
(1999); A. Brataas, Yu.V. Nazarov, J. Inoue, and G.E.W. Bauer, Eur. Phys. J. B 9, 421 (1999).

${ }^{9}$ A. Brataas, Yu.V. Nazarov, and G.E.W. Bauer, Phys. Rev. Lett. 84, 2481 (2000).

${ }^{10}$ Yu.V. Nazarov, Phys. Rev. Lett. 73, 1420 (1994); Yu.V. Nazarov, Superlattices Microstruct. 25, 1221 (1999).

${ }^{11}$ T. Valet and A. Fert, Phys. Rev. B 48, 7099 (1993).

${ }^{12}$ S.A. van Langen, H.P.A. Knops, J.C.J. Paasschens, and C.W.J. Beenakker, Phys. Rev. B 59, 2102 (1999).

${ }^{13}$ P.C. van Son, H. van Kempen, P. Wyder, Phys. Rev. Lett. 58, 2271 (1987).

${ }^{14}$ J. Rammer, Quantum Transport Theory, Frontiers in Physics Vol. 99 (Perseus Books, MA, 1998).

${ }^{15}$ B.H. Bransden and C.J. Joachain, Physics of Atoms and Molecules (Longman Group, Essex, 1983), p. 42.

${ }^{16}$ M. Büttiker, Phys. Rev. Lett. 57, 1761 (1986).

${ }^{17}$ A. Brataas, Yu. V. Nazarov, and G.E.W. Bauer, cond-mat/0006174 (unpublished).

${ }^{18}$ The energy dependence of $G^{T}$ is only relevant for energy scales of the order of the Fermi energy $E_{F}$, which is much higher than the revelant energy scale for transport, so we can consider $G^{T}$ as energy independent.

${ }^{19}$ R. Meservey and P.M. Tedrow, Phys. Rep. 238, 173 (1994).

${ }^{20}$ A.A. Abrikosov and L.P. Gor'kov, Zh. Éksp. Teor. Fiz. 42, 1088 (1962) [Sov. Phys. JETP 15, 752 (1962)].

${ }^{21}$ W. Kolbe, Phys. Rev. B 3, 320 (1971).

${ }^{22}$ Do not confuse the vector $\vec{\omega} \sim\left(\vec{m}_{\mathcal{L}} \times \vec{m}_{\mathcal{R}}\right)$ introduced in the Appendix, with the Larmor frequency vector $\vec{\omega}_{L}=\mu_{B} \vec{B} /$. 
${ }^{23}$ K. Tsukagoshi, B.W. Alphenaar, and H. Ago, Nature (London) 401, 572 (1999).

${ }^{24}$ L. Balents and R. Egger, cond-mat/0003038 (unpublished).

${ }^{25}$ M. Oestreich, J. Hubner, D. Hagele, P.J. Klar, W. Heimbrodt, W.W. Ruhle, D.E. Ashenford, and B. Lunn, Appl. Phys. Lett. 74, 1251 (1999); R. Fiederling, M. Keim, G. Reuscher, W. Ossau, G. Schmidt, A. Waag, and L.W. Molenkamp, Nature (Lon- don) 402, 787 (1999); Y. Ohno, D.K. Young, B. Beschoten, F. Matsukura, H. Ohno and D.D. Awschalom, ibid. 402, 790 (1999).

${ }^{26}$ G. Schmidt, L.W. Molenkamp, A.T. Filip, and B.J. van Wees, cond-mat/9911014 (unpublished).

${ }^{27}$ J.M. Kikkawa and D.D. Awschalom, Phys. Rev. Lett. 80, 4313 (1998). 